\title{
Philosophiques
}

\section{La recherche et le comité de déontologie à l'université}

\section{Georges Leroux}

Volume 5, numéro 1, avril 1978

URI : https://id.erudit.org/iderudit/203096ar

DOI : https://doi.org/10.7202/203096ar

Aller au sommaire du numéro

Éditeur(s)

Société de philosophie du Québec

ISSN

0316-2923 (imprimé)

1492-1391 (numérique)

Découvrir la revue

Citer ce document

Leroux, G. (1978). 3. La recherche et le comité de déontologie à l'université.

Philosophiques, 5(1), 200-208. https://doi.org/10.7202/203096ar d'utilisation que vous pouvez consulter en ligne.

https://apropos.erudit.org/fr/usagers/politique-dutilisation/ 


\title{
3. LA RECHERCHE ET LE COMITE DE DÉONTOLOGIE A L'UNIVERSITÉ
}

\author{
par Georges Leroux
}

\section{PRÉSENTATION}

Le texte qui suit constitue une intervention dans le débat suscité dans certaines institutions comme les universités, les centres de recherches et les hôpitaux, par la mise sur pied d'un Comité de déontologie. Ce Comité ne possède pas encore de prérogatives défi- nies et son existence est motivée, pour une bonne part, par le fait que plusieurs organismes subventionnaires ont jugé que le temps était venu de se munir de garanties morales avant de financer un projet de recherches.

L'Université du Québec a laissé à chacune de ses constituantes la tâche de créer un tel Comité, habilité à donner des avis aux organismes subventionnaires. Elle a également travaillé à préciser la problématique et à définir les principes de la déontologie de la recherche. C'est à titre de professeur à l'UQAM que j'ai participé aux discussions d'un groupe de travail dont le mandat était dé formuler des suggestions pour l'élaboration d'une politique générale en matière de déontologie de la recherche. J'y ai côtoyé des chercheurs provenant de plusieurs disciplines, en plus d'un juriste et d'un théologien, et j'ai beaucoup appris dans nos discussions. Compte tenu du contexte dans lequel fut rédigé le texte que je soumets ici aux critiques de collègues philosophes, je m'excuse à l'avance de son caractère essentiellement aporétique. Il vient de ce que partout où je croyais pouvoir énoncer une proposition claire et ferme d'un point de vue philosophique, on a fait valoir des objec. tions tirées de la pratique scientifique dont $j$ 'avais mésestimé la pertinence.

\section{RECHERCHE ET DÉONTOLOGIE}

Dans la discussion des prérogatives d'un Comité universitaire de déontologie de la recherche, deux problèmes essentiels doivent 
être abordés. Il y a d'abord le rapport étroit de l'éthique de la recherche à l'ensemble du contrat social auquel soucrit l'Université ; ce rapport est tel qu'on doit chercher à éclaircir la question de savoir si la recherche peut encore être dite libre de ses conséquences prévisibles à tous les plans où celles-ci peuvent se manifester. L'institutionnalisation progressive de la recherche dans des centres soutenus directement par l'État donne à cette première question un contexte fondamentalement politique : l'Université peut-elle développer une éthique de la recherche qui ne tienne pas compte des directions que voudrait lui imprimer le public qui la subventionne? Peut-elle le faire sans nuire à la liberté de la recherche, à son dynamisme intrinsèque ? Cela introduit le second facteur déterminant dans l'analyse des prérogatives d'un Comité de déontologie: la liberté du chercheur, en particulier dans ses aspects heuristiques et organisationnels. Cette liberté est en effet traditionnellement liée au développement interne de l'observation et de la conceptualisation, mais elle comporte en outre aujourd'hui des dimensions liées à la complexification des procédures d'expérimentation et des modes de subvention, dont l'importance ne saurait être mésestimée. S'il est difficile de concevoir qu'un Comité de déontologie puisse intervenir en contraignant la liberté heuristique d'une recherche, il est fréquent de constater que ce Comité peut entraver, pour cause, l'organisation et le financement d'une recherche.

La mise sur pied de Comités institutionnels de déontologie répond clairement à la nécessité de régler le développement de la recherche sur le plan de ses conséquences. Une telle préoccupation s'exerce d'abord en ce qui a trait à la protection des sujets humains de l'expérimentation, mais elle intervient également au niveau des objectifs globaux d'une société, dont la détermination n'appartient pas au seul développement de la science mais encore à l'exercice d'une volonté politique. La question qui se pose alors est la suivante: l'institution peut-elle tenter de régler la recherche sur le plan de ses conséquences sans intervenir sur le plan de ses hypothèses? La vigilance éthique d'une société peut-elle s'exercer sans entraver le développement complexe du savoir?

La réponse à cette question n'est pas simple à fournir et de nombreux débats, concernant notamment ce qu'il est convenu d'appeler l'idéologie de la science, n'ont pas encore permis d'en donner même une claire formulation. On ferait cependant un pas 
en avant si on consentait à voir dans l'apparente contradiction entre les contraintes toujours plus grandes exercées par l'institution et la liberté de la recherche un problème plus moral que logique. L'institution de Comités de déontologie demeure en effet doublement limitée. Le mandat en est restreint aux conditions de l'expérimentation ; cela découle du fait que les conséquences prévisibles d'une recherche demeurent toujours moralement indéterminées et que seul l'usage du savoir peut être moralement déterminé, c'est-àdire soumis à un jugement de valeur. Le Comité de déontologie limite donc son expertise à l'examen des seules conditions de l'expérimentation pouvant avoir des effets néfastes pour des sujets participants ou encore des conditions soustraites à la connaissance des sujets et établies sans leur consentement. En second lieu, le Comité de déontologie limite son mandat à l'examen des seuls projets de recherche mis de l'avant dans l'institution. Il demeure sans prise sur la recherche privée. En conséquence, l'adhésion du chercheur à l'institution constitue, de facto, une adhésion à un système de principes qui est celui de l'institution et qui tient compte des normes de la société qui supporte cette dernière.

On ne saurait donc faire valoir, au moins dans l'état actuel de la politique de la science, - caractérisé par une grande timidité, - que l'institution de Comités de déontologie entraîne une limitation théorique ou pratique de la liberté de la recherche. Elle ne fait au contraire, dans la pratique, comme en témoigne le Rapport du Comité de déontologie du Conseil des Arts du Canada ${ }^{1}$, que rappeler aux chercheurs les conditions minimales de la moralité de l'expérimentation avec des sujets humains. Elle ne leur dit rien de l'éthique de la science, encore moins de la politique sociale. Le Comité de déontologie rappelle l'appartenance à l'institution, il n'énonce pas les rapports de la recherche à la société. On ne peut donc lui reprocher de durcir l'opposition entre le contrôle institutionnel et la liberté de la recherche, puisqu'il n'exerce de contrôle

1. Déontologie: Rapport du Groupe consultatif de déontologie. Conseil des Arts du Canada, 1977. Ce Rapport concerne surtout la recherche en sciences humaines, par exemple sur les enfants, sur les prisonniers, etc. Le mandat des Comités institutionnels est discuté aux pages 28-30. Pour le domaine bio-médical, on pourra consulter le Rapport du Conseil de Recherches médicales intitulé La déontologie de l'expérimentation chez l'bumain, Ottawa, 1978 (Rapport no 6). Le Rapport contient, à la p. 55 et ss, une bibliographie d'orientation très utile, dans laquelle on trouvera à la fois les documents internationaux (par ex. le code de Nuremburg) et la bibliographie générale. 
que sur la recherche de l'institution même, et encore ne le fait-il que minimalement.

Cette première conclusion porte notre question à un niveau proprement éthique: l'adhésion du chercheur à l'institution entraîne-t-elle pour lui le devoir de se conformer à ses règles? Il est important de dire ici qu'une réponse affirmative à cette question pourrait n'avoir guère de conséquences, tant que l'institution ne veut que contrôler les conditions de l'expérimentation, mais qu'elle pourrait en avoir de considérables si l'institution décidait, par exemple sous la pression de l'opinion, de régir le développement même de la recherche, par exemple en discriminant les projets à partir d'objectifs de nature purement sociale, politique, militaire, etc . . La composition du Comité de déontologie deviendrait alors une question fort complexe, puisque deux avenues, et deux seulement, s'offriraient à l'institution universitaire et de recherche : l'autonomie et la servilité. C'est ici qu'intervient, à proprement parler, la liberté du chercheur : liberté de ne pas souscrire à l'institution si elle est servile, liberté de s'en détacher si, étant autonome, l'institution contredit néanmoins les principes moraux du chercheur. On voit donc que la moralité de l'adhésion du chercheur à l'institution est plus difficile à éclaircir dans le cas d'un contrôle étendu que dans le cas, actuel, d'un contrôle minimal. S'il est difficile d'asservir le chercheur à l'institution, dans le cas de la détermination des objets même de la recherche, il est concevable de l'y soumettre pour la seule détermination des conditions de cette recherche.

Tel est l'objectif d'un Comité de déontologie, telle est sa prérogative élémentaire. Ultimement, celle-ci découle des contraintes qui ne peuvent manquer de s'exercer sur la recherche institutionnalisée. Le concept de la liberté de la recherche lui-même est discuté, car il autorise une position facilement admise en principe, mais qui rencontre plusieurs difficultés sur le plan de la pratique. Ce qui est admis en principe l'est aussi généralement : une recherche qui contredirait manifestement le bien-être des sujets participants, qui contribuerait à dégrader un environnement ou qui aurait des conséquences clairement néfastes sur les individus ou les lieux qui en subiraient l'expérimentation, ou une recherche dont les conditions seraient camouflées aux sujets participants ne saurait être entreprise sans contrevenir à l'éthique la plus fondamentale 
d'une société. Cette situation ne se présente jamais sur le plan des objets d'une recherche, puisque les projets de recherche possèdent en général les caractéristiques suffisantes pour garantir leur moralité, à proportion de la visée d'amélioration qu'ils proposent. Même dans le cas de la recherche dite fondamentale, le rapport de cette recherche à ses applications peut toujours être énoncé de manière ambiguë : les utilisations positives pourront toujours compenser, au moins quant aux prévisions, les utilisations négatives qui donneraient lieu à la réserve éthique. Une telle ambiguïté est sans doute suffisante pour dissoudre en son principe jusqu'à l'idée d'une éthique de la science : aucun savoir n'est moralement déterminé. En conséquence, sur le plan de ses objets, un principe admis dans sa généralité, - à savoir que la science ne saurait s'engager sur des chemins qui manifestement détruisent la société ou l'environnement, - peut ne recevoir jamais aucune application. Le rapport entre un projet de recherche et ses conséquences n'est en effet jamais manifeste, mais toujours implicite, toujours ambigu. ${ }^{2}$

Le premier seuil où cette ambiguïté peut être levée est celui des conditions de la recherche ; c'est là que la contrainte de l'Université et des organismes subventionnaires sur la direction de la recherche, en fonction de la société qui la supporte, doit d'abord s'exercer. La prévisibilité des conditions de la recherche doit être assez précise pour garantir leur moralité : voilà ce qu'exige en un premier temps l'institution. On doit donc pouvoir en donner une description assez concrète et dépourvue d'ambiguïtés. C'est à cette tâche que contribuent les codes et procédures de déontologie visant à protéger immédiatement les personnes impliquées directement dans l'expérimentation.

Ce principe une fois admis peut-on soutenir qu'il contredit la liberté académique? Nous ne le pensons pas. L'application de ce principe entraîne que tout projet de recherche qui a des conséquences prévisibles ayant une incidence éthique doit être soumis au Comité de déontologie de l'Université. L'universalisation de cette application doit être maintenue fortement, même si le chercheur peut alors arguer des retards encourus par ce processus. Cet aspect de la discussion est organisationnel.

2. C'est à lever cette ambiguïté dans tous les cas particuliers où il est possible de le faire que travaillent les collaborateurs du Hastings Center, dans l'État de New York. Les publications de ce Centre (Hastings Centre Report) portent le plus souvent sur les implications éthiques de recherches particulières. 
On ne saurait en effet, dans le domaine de l'éthique, faire valoir une contrainte de temps contre un principe moral. S'il est certain que la contrainte de temps vient à bout de la moralité des meilleurs chercheurs, il n'en est que plus clair qu'un Comité de déontologie doit tenir pour nuls les arguments de ceux qui, au nom de la liberté académique, refusent l'examen du Comité, en mettant de l'avant la question administrative. ${ }^{3}$

Cette tâche est limitée, elle représente un mandat restreint tout en constituant pour le chercheur une obligation morale forte. Il est sans doute à prévoir qu'elle se développera, en un second temps, dans le sens d'une précision accrue de toutes les conséquences d'un projet de recherche susceptibles d'intéresser une discussion éthique. S'il est vrai que le principe d'une éthique de la science demeure non fondé sur le plan de la prévision, il peut sans doute être établi en regard d'une optimalisation des résultats bénéfiques. Déjà en effet la tâche et le devoir de rendre explicites les conséquences virtuelles d'un projet établissent les conditions suffisantes de sa moralité. Ceci est sans doute le prochain seuil que devra atteindre le Comité de déontologie : non pas la prévision du bénéfique, qui demeure ambiguë, mais l'énoncé de toutes les possibilités et la description du probable. La liberté de la science et de la recherche reçoit à ce niveau une détermination positive qui ne peut en contredire le concept : le Comité de déontologie ne discrimine pas les projets selon leurs implications prévisibles, il n'a pour tâche que de veiller à stimuler l'énoncé de ces implications. Il appelle à la vigilance éthique les chercheurs, il ne se substitue pas à eux.

Le troisième temps ou seuil d'intervention d'un Comité de déontologie demeure encore si éloigné qu'on répugne à l'évoquer ; il implique en effet un état de la société où le contrôle de la science n'appartient ni à ceux qui l'exercent - ne répondant en conséquence à aucun critère interne de développement, — ni à ceux qui tiennent une position dans la vie politique, mais à une instance autonome, reconnue à la fois par les chercheurs et par l'institution sociale et en conséquence susceptible de contraindre les premiers au nom d'un contrat social inédit. On ne voit pas, malgré l'obscurité qui s'attache à cette hypothèse, qu'on puisse éviter d'y parvenir ; c'est pour cette raison qu'on la mentionne ici.

3. BARBER, Bernard, "The Ethics of Experimentation with Human Subjects ", Scientific American 234 (1976) 25-31. 
En effet, aucune des positions représentées dans l'histoire des sciences en ce qui a trait à l'orientation de la recherche n'est satisfaisante. Cette orientation ne peut être laissée à l'État sans ouvrir la porte à un contrôle, dont un exemple aberrant fut le lyssenkisme. Une telle position, qui exclut en quelque sorte les chercheurs, demeure intenable. Inversement, des travaux récents et de plus en plus nombreux tendent à montrer l'anarchie et le chaos d'un développement de la recherche livré aux seules lois de la compétition et de la technologie. La société actuelle est donc mise au défi d'articuler un mécanisme satisfaisant pour empêcher le contrôle étatique, tout en réglant l'anomie de la recherche à l'ère industrielle.

Pour progresser dans cette voie, il faudra d'abord résoudre un problème actuellement débattu : les rapports de la science et de l'idéologie. Le chercheur peut en effet faire valoir que le principe de la soumission de la recherche aux fins d'une société est idéologiquement déterminé, et ce, même dans les cas où les droits de la personne sont engagés. Par exemple, quelqu'un pourrait soutenir que si une expérience devait avoir un risque élevé de mortalité, les transplantations d'organes, - et néanmoins garantir une brèche dans la direction de la solution des problèmes de ce genre, alors elle devrait néanmoins être tentée. Cette personne présuppose alors que le concept même de "personne " est idéologiquement déterminé. De manière plus concrète, on pourrait faire valoir qu'une recherche met souvent en cause une institution politique (par exemple, les recherches sur la psychologie de l'intelligence dans des races distinctes). ${ }^{4}$

Ce dernier aspect fait resurgir ce qui est actuellement évacué : le conflit entre la liberté du chercheur et la soumission à un Comité de déontologie; en effet, les principes régissant l'examen d'un projet sont eux-mêmes l'expression d'idées déterminées, possiblement réfutables, etc...

Le cas du professeur HORN, à qui on refusa des subventions qui lui étaient nécessaires parce que ses recherches mettaient en cause la psychologie des enfants noirs américains, en particulier le caractère héréditaire de l'intelligence, est bien instructif à cet égard. Il ne fait aucun doute que les Comités de déontologie

4. HORN, L. John, "The Ethics of Research : A Case Study and its Lessons ", in S. HOOK, P. KURTZ \& M. TORODOVITCH, Eds. The Etbics of Teaching and Scientific Research, New York, Prometheus Books, 1977, p. 135-159. 
peuvent abuser de leur pouvoir de contrôle dans ce type de recherches. L'exemple en cause montre bien cependant le paradigme des recherches qui posent ce problème : il s'agit de recherches idéologiquement orientées. C'est en ce sens qu'on cite Galilée et Copernic à l'appui de sa position quand on sent qu'on veut soi-même ébranler une conception du monde. A vrai dire, le paradigme de la science libre appartient au passé révolu de la science; dans le présent, il ne convient peut-être qu'à la philosophie qui est idéologiquement plus déterminée que les sciences. Aucune science ne partage entièrement ce prédicat avec la philosophie, et les chercheurs qui refuseraient l'examen du Comité de déontologie, pour la raison que cet examen pourrait être idéologiquement biaisé, ne font que révéler par là même qu'il se trouve dans leurs préoccupations quelque chose qui donne prise à un tel biais. Dans l'état actuel cependant, ils pourraient avoir raison : les comités prévus n'ont d'autre seuil d'intervention clair que celui des conditions de l'expérimentation.

L'éventualité problématique d'une instance chargée de régir le développement de la recherche préjuge, en effet, de la possibilité de distinguer dans un projet son orientation idéologique particulière. Comme aucun moyen satisfaisant n'est à notre disposition pour fournir des analyses rigoureuses à ce chapitre, il faut donner comme consignes au comité d'éthique de limiter les critères de son txamen à des critères identifiables et précis. Dans un premier temps, et cela pourra rassurer tous les chercheurs, ces critères doivent demeurer limités au bien-être des participants à l'expérimentation, à la qualité de l'environnement physique, à la confidentialité des informations, etc., bref, à des critères qui n'interfèrent en aucune façon avec l'heuristique de la recherche en cause. Ce problème, plus délicat, sera laissé à une étape ultérieure, dans laquelle seront définis les critères éthiques d'une recherche, au niveau même de son heuristique. Notre position exprime un vœu, correspondant à la nécessité d'une situation, mais il se pourrait que ce væu ne soit jamais réalisable.

Il faut donc donner au Comité de déontologie une fonction destinée à se développer proportionnellement aux risques de l'expérimentation scientifique, aux manipulations des sciences humaines et au pouvoir qui en découle pour les utilisateurs de ces connaissances. C'est en vertu du principe que la science n'est possible 


\section{que par la société qu'on doit argumenter pour le principe qu'elle doit être faite aussi pour elle, et non contre elle.'}

5. On consultera avec profit le recueil édité par les soins de P.A. FREUND : Experimentation with human subjects, New-York, Braziller, 1969 (Dædalus Library). Ce livre contient, entre autres, des textes de H. Jonas, T. Parsons, M. Mead. Nous n'avons pas mentionné le problème, très discuté chez les philosophes, des droits des animaux ; cf. S.R.L. CLARK : The Moral Status of Animals, Oxford, 1977. Il faut signaler le développement récent de l'éthique bio-médicale ; H.T. ENGELHARDT et S.F. SPICKER dirigent, chez l'éditeur Reidel, une collection intitulée "Philosophy and Medicine ", déjà très riche. Notons également la publication à l'Université de Chicago, depuis mars 1976, d'un périodique spécialisé dans ces questions: The Journal of Medecine and Pbilosopby. Enfin, il faut attirer l'attention sur la création à Montréal, dans le cadre de l'Institut de Recherches cliniques, d'un Centre de Bioéthique dirigé par un théologien, M. David Roy. 